\title{
Challenges in Urology during the COVID-19 Pandemic
}

\author{
Peng Jin ${ }^{a, b}$ Hyusim Park ${ }^{c}$ Sungyong Jung ${ }^{c}$ Jayoung Kim ${ }^{a, d}$

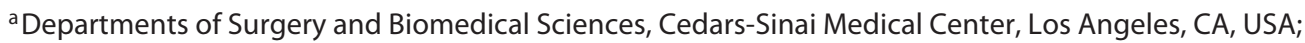 \\ ${ }^{b}$ Department of Urology, Shengjing Hospital of China Medical University, Shenyang, China; ${ }^{C}$ Department of \\ Electrical Engineering, University of Texas at Arlington, Arlington, TX, USA; ${ }^{d}$ Department of Medicine, \\ University of California, Los Angeles, CA, USA
}

\section{Keywords}

COVID-19 pandemic - Test methods - Clinical challenges .

Urological diseases · Urologists

\begin{abstract}
The COVID-19 pandemic has caused a global health threat. This disease has brought about huge changes in the priorities of medical and surgical procedures. This short review article summarizes several test methods for COVID-19 that are currently being used or under development. This paper also introduces the corresponding changes in the diagnosis and treatment of urological diseases during the COVID-19 pandemic. We further discuss the potential impacts of the pandemic on urology, including the outpatient setting, clinical work, teaching, and research.

(c) 2020 S. Karger AG, Basel
\end{abstract}

\section{Introduction}

Coronaviruses are enveloped, positive-sense, and single-stranded RNA viruses that can be subdivided into 4 different classes, i.e., $\alpha, \beta, \gamma$, and $\delta$. In recent years, sev- eral coronaviruses have caused epidemics in various regions of the world (in 2002-2003 there was the SARS-Cov epidemic in China and in 2012 there was the MERS-CoV epidemic in Saudi Arabia). The appearance of the new viral SARS-CoV-2 (COVID-19) strain in 2019 that originated in the Chinese region of Wuhan started a global pandemic, afflicting over a million people and causing over 50,000 deaths [1]. COVID-19 belongs to the $\beta$-coronavirus family, and it is thought to have originated in bats. There is a noted similarity between the genomic sequence of human COVID-19 and that of HKU9-1 in bats; however, the intermediate host between bats and humans has yet to be identified. COVID-19 is highly contagious and it has 3 main routes for transmission, i.e., person-to-person contact, aerosol, and touch. In addition to infecting the respiratory system, the virus also infects the blood, digestive, and urinary systems. As a result, the presence of the virus has been detected in fecal, blood, and urine samples. COVID-19 can cause a variety of symptoms, such as fever, dry cough, shortness of breath, loss of appetite, and fatigue. Furthermore, hidden transmission can occur from asymptomatic individuals. The incubation period for COVID-19 ranges between 2 and 14 days. karger@karger.com www.karger.com/uin

(c) 2020 S. Karger AG, Basel

Karger"
Jayoung Kim

Departments of Surgery and Biomedical Sciences Cedars-Sinai Medical Center, Davis 5071 8700 Beverly Blvd., Los Angeles, CA 90048 (USA) Jayoung.kim@csmc.edu 
How will life change during and after the COVID-19 pandemic from the point of view of a urologist? In this short article, we will discuss the COVID-19 pandemic and its effects on the field of urology.

\section{Discussion}

\section{COVID-19 Examination}

\section{Common Test Methods}

The general structure of coronaviruses includes spike glycoproteins, membrane proteins, nucleoproteins, and genomic RNA [2]. To detect the novel SARS-CoV-2, nucleic acid testing is the main technique for laboratory diagnoses [3]. Sample sources include sputum, throat swabs, and lower respiratory system secretions. Other methods, such as virus antigen or serological antibody testing, are valuable assays for detecting infection. The main sample source for these tests is blood. As with other emerging viruses, the development of methods was only able to begin after identification of the viral genome.

Real-time reverse transcription-polymerase chain reaction (RT-PCR) has become the standard method for diagnosing COVID-19 due to its specificity, sensitivity, and simplicity. Zhang et al. [4] used RT-PCR to detect COVID-19 from throat and rectal swab samples from patients. The virus was detected in both types of samples. Throat swabs are suggested to be more applicable in the early stages of infection, while rectal swabs are better for detecting late-stage infections [4].

Unfortunately, RT-PCR detection kits have many limitations, such as long turnaround times, complex operational procedures, expensive equipment, technical personnel requirements, false negatives, and so on [2]. As a result, these limitations make RT-PCR unsuitable for rapid and simple diagnosis and screening of patients $[5,6]$. On the other hand, the COVID-19 IgG-IgM combined antibody test can generate results within $15 \mathrm{~min}$ and it can determine whether the viral infection occurred recently. It is easy to use, has a high sensitivity, and does not require additional equipment [4]. This rapid detection method has great potential benefits for the mass screening of $\mathrm{CO}$ VID-19 infections [7, 8].

The COVID-19 IgG-IgM combined antibody test kit detects antibodies in the blood that are produced by the body's immune system for a prolonged period of time after infection. However, further studies of IgG-IgM tests are needed in order to validate that it provides a high enough specificity and sensitivity to be widely used [9]. RT-PCR has more benefits when diagnosing patients ini- tially, while IgG-IgM is very useful in monitoring confirmed patients or identifying late-stage patients for the prevention of spread. As of May 17, 2020, there were 75 different COVID-19 test kit products that were Emergency Use Authorization (EUA) approved [9].

Table 1 shows a list of clinically evaluated test kits approved by EUA with results from positive percent agreement and negative percent agreement.

\section{Routine Blood Test for COVID-19}

COVID-19 can cause serological abnormalities. Some studies have shown that the levels of lymphocytes, platelets, hemoglobin, and albumin in COVID-19 patients are decreased while levels of glucose, lactate dehydrogenase, interleukin-6, serum ferritin, C-reactive protein, ESR, alanine aminotransferase, aspartate aminotransferase, creatine kinase, $\mathrm{D}$-dimer, and serum procalcitonin are increased $[10,11]$. However, these routine serological examinations can only indicate infection and have poor specificity, which means that they cannot be used to confirm any diagnoses of COVID-19.

\section{Expression of ACE2 and TMPRSS2 in the Urinary} System and COVID-19

The human angiotensin-converting enzyme 2 (ACE2) gene is located on chromosome Xp22 and includes 18 exons [12]. Functioning as a typical zinc metallopeptidase, the ACE2 protein contains 805 amino acids and is a type I integral membrane glycoprotein containing a single catalytic domain. In the renin-angiotensin system, ACE2 degrades angiotensin II, which is potent in vasoconstriction, proinflammation, and pro-fibrosis, converting it into Ang (1-7) which contributes to vasodilation, antiproliferation, and apoptosis. Besides its systemic effects on blood pressure regulation, ACE2 also regulates amino acid absorption in the kidneys and gut and modulates the expression of amino acid transporters.

COVID-19 has a specific spike protein 3-D structure that is characterized by a strong binding affinity to ACE2 receptors [13]. As a result, human cells that express ACE2 may act as target cells for COVID-19. Since COVID-19 must bind with ACE2 first before entering the human host cells, the distribution and expression of ACE2 may be critical for the target organ of infection [14]. It is known that ACE2 is enriched in the heart, kidneys, and testes, and it is also broadly distributed in the lungs, liver, intestine, and brain [15]. Therefore, COVID-19 mainly infects the respiratory system. In addition to respiratory symptoms, the common complications found in patients infected with COVID-19 include acute cardiac and kidney injuries. 
Table 1. EAU-approved COVID-19 test kits

\begin{tabular}{llll}
\hline Test kit & Detection type & Manufacturer & PPA (\%)/NPA (\%) \\
\hline Xpert Xpress SARS-CoV-2 test & RT-PCR & Cepheid & $98.3 / 100[105]$ \\
\hline ePlex SARS-CoV-2 & RT-PCR & GenMark Diagnostics & $91.4 / 100[105]$ \\
\hline Allplex ${ }^{\text {TM }}$ 2019-nCoV & & & $94.4 / 100[106]$ \\
\hline Anti-SARS-CoV-2 rapid test & RT-PCR & Seegene & $100 / 94[107]$ \\
\hline
\end{tabular}

The transmembrane serine proteinase 2 (TMPRSS2) gene was first identified on human chromosome 21 in 1997 [16]. The full-length cDNA encodes a predicted 492 amino acid protein, which is anchored to the plasma membrane and belongs to the TTSPs family (type II transmembrane serine proteases). In humans, TMPRSS2 is mainly expressed on prostate, pancreatic, and colon cells, but it can also be found in lung, liver, and kidney cells [17]. The entry of SARS-CoV-2 into host cells depends on the serine protease activity of TMPRSS283-87. Cells overexpressing TMPRSS2 are susceptible to SARSCoV-2 infection [18]. The process by which SARS-CoV-2 enters the host cell can be divided into 2 steps. In the first step the viral hemagglutinin protein attaches to ACE-2, and in the second step the hemagglutinin is cleaved to activate the internalization of the virus. This second step depends on the expression of TMPRSS2 [19].

\section{Fecal/Urine/Semen Test for COVID-19}

COVID-19 is primarily transmitted through the respiratory tract [19]. However, ACE2 expression patterns across different tissue types suggest the possibility of extrarespiratory viral transmission through bodily fluids [20]. The current focus has been placed mainly on viral clearance from respiratory secretions and little is known about the possible concurrent presence and clearance through bodily fluids.

At present, the virus is mainly detected by nasopharyngeal/oropharyngeal swabs. In addition to nasopharyngeal/oropharyngeal swabs, the presence of COVID-19 RNA has also been reported in fecal, urine, and blood samples. Feces appear to contain a high percentage of viral RNA, and the percentage of patients with viral RNA in urine and blood appears to be low. However, some studies have been contradictory; no COVID-19 RNA was found in the urine of infected patients [21].

Although this represents pertinent information related to reproductive medicine, the presence of COVID-19 in semen has not been investigated [21]. There have been few studies investigating the presence of SARS-CoV-2 in semen, and no solid reports on virus presence in semen are available. The small studies that have been done have found no viruses in semen. The sample sources came from patients with acute infection and convalescence, as well as testicular biopsies from deceased patients $[21,22]$. Moreover, a recent paper published in August 2020 showed no COVID-19 viral transmission during sexual contact or assisted reproductive techniques [23]. Only one study detected the presence of SARS-CoV-2 in semen; however, the sample size was small and the possibility that the virus came from urine could not be ruled out [24]. The virus may persist in the prostate or urethra and be carried away by semen during ejaculation. Moreover, patients with severe COVID-19 infection may have contaminated specimens when collecting semen. So far, there has been no report of SARS-CoV-2 RNA in the expressed prostatic secretions of COVID-19 patients [25].

\section{Gender and COVID-19}

So far, most countries with available data have reported that men with COVID-19 have a greater severity of illness and a higher mortality rate than women across all age groups [26]. Possible reasons for this disparity include gender-related differences in ACE2 receptors, immune function, sex hormones, hygiene, habits, etc. [27]. In previous cases of pathogenic coronaviruses, this difference between men and women also existed. In the 20022003 SARS outbreak and the 2012 MERS outbreak, the mortality rate was comparatively higher in men [28].

The relationship between COVID-19 and ACE2 has been described before. Studies have shown that men have more ACE2 receptors than women and they have a greater expression of ACE2 in the lungs and heart, which may explain why men tend to have more serious disease [29]. Differences in immune defense abilities may also lead to differences in COVID-19 outcomes between men and 
women. Compared to males, females usually produce more robust innate and adaptive immune responses and respond more strongly to most invading pathogens [30].

Sex hormones play a role in regulating the immune system and they can cause differences in immune response between men and women. Generally speaking, testosterone has an immunosuppressive effect, while estrogen tends to enhance the immune response [31]. Endogenous testosterone makes men more likely to have more serious complications related to SARS-CoV-2 infection. On the other hand, SARS-CoV-2 infection can lead to hypogonadism in men, and the reduction of androgens may cause serious complications [32]. Given that estrogen activates the immune system, higher estrogen levels can potentially have a protective effect [33]. Studies have shown that exogenous estrogen therapy can eliminate inflammation and reduce virus titers, thus improving survival [34]. The differences in hygiene and habits provide another explanation for the gender-related differences in SARS-CoV-2 infection. Studies have shown that women wash their hands more frequently than men [35], and men wear masks less than women [36]. These factors may explain the noted differences in COVID-19 rates and mortality between the sexes.

The higher smoking rate among men may also be responsible for gender-related differences in COVID-19 mortality. Smokers seem to have a higher risk of respiratory infections. A study found that the expression of ACE2 in Asian smokers is significantly higher than that in nonsmokers [37]. However, the current literature does not support smoking as a predisposing factor for SARS$\mathrm{CoV}-2$ infection. Gender differences are also reflected in men's underutilization of medical services and participation in unhealthy habits, such as alcohol use and smoking. These behaviors can cause a high blood pressure, cardiovascular disease, and other comorbidities associated with increased mortality from SARS-CoV-2. Because cells overexpressing TMPRSS2 are susceptible to SARS-CoV-2 infection, and the expression of TMPRSS2 is regulated by androgen/androgen receptor (AR) signals, the expressions of AR and TMPRSS2 are significantly positively correlated [38]. This may be a reason why there are more male patients than female patients [19].

Assisted Reproductive Technology and COVID-19

In light of the current COVID-19 pandemic and the uncertainty of its impact on pregnant women, the Society of Human Reproduction published recommendations for the management of patients undergoing infertility treatment through assisted reproductive technology
(ART) [39]. ART includes intrauterine insemination, in vitro fertilization (IVF) injection, ovum collection, implantation, transplantation, and tracking. This process could expose infertile individuals to COVID-19 infection [40]. In addition to the risk of infection, the World Health Organization (WHO) published data showing that pregnant women with COVID-19 are at greater risk of serious complications [41]. There are no clear answers as to whether there is a risk of vertical transmission of COVID-19 from mother to fetus. At present, no such cases have been reported. In a case in China, newborns were infected with the SARS-CoV-2 virus $36 \mathrm{~h}$ after birth [42]. However, it is unclear whether this was due to vertical transmission between mother and child [42].

At present, most reports indicate that the symptoms of pregnant women infected with COVID-19 are similar to those of others, and there is no evidence showing that pregnant women or fetuses are at a higher risk [43]. However, considering the initial data and the lack of comprehensive understanding of the pathogenesis of SARSCoV-2 during pregnancy, most human reproduction societies have proposed postponing embryo transfer and delay of new treatment cycles.

The European Society of Human Reproduction and Embryology (ESHRE) issued a statement regarding COVID-19 and pregnancies on March 14, 2020. So far, only a few cases of COVID-19 infection during pregnancy have been reported. Therefore, there is not enough information available about the potential impact of COVID-19 infection in the early stages of pregnancy. Additionally, drug treatment for severe COVID-19 cases may include drugs that are dangerous or prohibited for use during pregnancy. The ESHRE recommends that all patients considering or planning to receive ART treatment, regardless of whether they are diagnosed with or suspected to have COVID-19 infection, should avoid pregnancy at this time and consider delaying pregnancy by freezing oocytes or embryos to delay embryo transfer [44].

In the latest guidelines updated in October 2020, the ESHRE divides the guidelines for ART services during a viral pandemic into 2 core steps. The first step is to assess the impact of the current pandemic based on epidemiological factors. The second step is to take corresponding measures based on this assessment [45]. See Table 2 for specific guidelines.

On March 17, 2020, the American Society for Reproductive Medicine (ASRM) released a new document titled "Patient Management and Clinical Recommendations during the Coronavirus (COVID-19) Pandemic" [46]. The main recommendations are as follows: 
Table 2. ESHRE guidance for safe ART services during the third phase of the COVID-19 pandemic

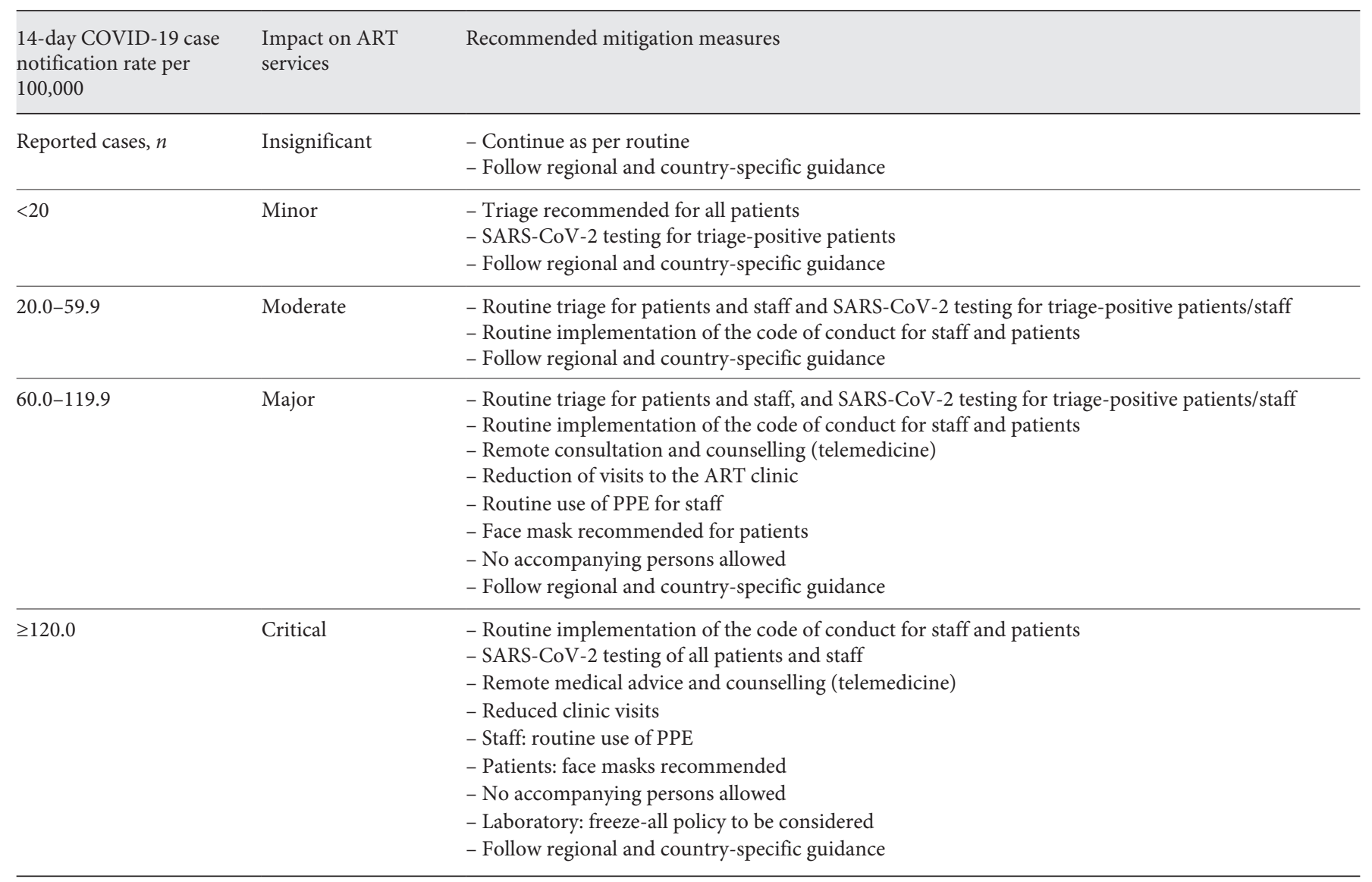

- Suspend the start of new treatment cycles, including ovulation induction, intrauterine insemination, IVF, retrieval and frozen-embryo transfer, and nonemergency gamete cryopreservation.

- Strongly consider canceling all embryo transfers, whether fresh or frozen.

- Continue to care for patients who are currently "in cycle" or in need of emergency stimulation and cryopreservation.

- Suspend the selection of surgery and nonemergency diagnostic procedures.

- Minimize interpersonal interaction and increase the utilization of telemedicine.

In June 2020, the ASRM published the fifth update of patient management and clinical recommendations during the viral pandemic [47]. For details, please refer to Table 3.

However, each organization has also stressed that treatment should be carried out in certain cases, such as those in which women are required to retain fertility due to tumor causes and in cases where delayed treatment may be more harmful than continued treatment (i.e., patients with a low ovarian reserve).

During the pandemic, the IVF department should encourage male patients to collect semen samples at home and send them to the laboratory. However, semen samples must also be considered as potential sources of infection during this process. Assessment of the presence of SARS-CoV-2 in semen is particularly important for semen cryopreservation because storage in liquid nitrogen retains the pathogenic properties of the virus [48]. Therefore, it is necessary to conduct large-scale studies on currently infected patients to confirm or exclude the risk of male gametes. These risks need to be assessed for cryopreservation in liquid nitrogen or ART. It should be noted that a vast majority of published reports so far suggest that there is no SARS-CoV-2 in semen. Only 1 article mentioned the presence of the virus in semen, but the report itself has some questionable factors. 
Table 3. ASRM patient management and clinical recommendations during the coronavirus (COVID-19) pandemic: 5th update

\begin{tabular}{ll}
\hline Third-party reproduction & $\begin{array}{l}\text { Do not start a cycle where the intended parents and the pregnant carrier do not live in the same } \\
\text { country. }\end{array}$ \\
\hline $\begin{array}{l}\text { Fertility services for } \\
\text { healthcare workers }\end{array}$ & $\begin{array}{l}\text { Take routine precautions; there is no data to support the need for medical staff to avoid infertility } \\
\text { treatment or pregnancy. }\end{array}$ \\
\hline $\begin{array}{l}\text { Having partners present } \\
\text { when providing care }\end{array}$ & $\begin{array}{l}\text { The number of people in the ward must be limited, and partners are encouraged to use telephone or } \\
\text { video methods to participate in the escort. }\end{array}$ \\
\hline Travel restrictions & $\begin{array}{l}\text { Where feasible, avoid using public transportation; if there is an emergency trip, you need to know the } \\
\text { new infection rate in the local area and destination; self-isolation should continue to be practiced as } \\
\text { much as possible. }\end{array}$ \\
\hline
\end{tabular}

Resumption of reproductive surgery

Reproductive surgery can be resumed in areas where the prevalence of the disease should be low $(<12 \%)$. Before any surgery that requires anesthesia, the patient should be checked for COVID-19 symptoms. Preoperative SARS-CoV-2 virus detection should also be strongly considered. If the virus test is positive, the patient needs to be rechecked for negativity before scheduling surgery.

Aerosol-generating procedures

If aerosol-generating procedures must be performed urgently during the operation, except for the anesthesiologist and the technical or circulatory nurse, the operation team should consider leaving the room immediately. Staff can wear appropriate, fully enhanced PPE. These precautions should be taken regardless of whether the patient has a negative preoperative test result, is asymptomatic, or has no test result at all.

IVF cycles During the oocyte retrieval process, all patients should wear medical-grade surgical masks. Any planned embryo transfer should be delayed until the patient is SARS-CoV-2 negative.
The ASRM and the ESHRE provide differing opinions on whether to perform SARS-CoV-2 testing on patients and providers [49]. The ASRM points out that, based on existing evidence, nucleic acid amplification testingbased testing should be considered before reproductive surgery or other aerosol-generating procedures. However, for antibody testing, ASRM believes that antibody testing should not be used in the decision-making of patients or providers, and it should not change compliance with personal protective equipment (PPE) guidelines. On the other hand, ESHRE relies more on serological testing. It is recommended that, if either party develops symptoms within 2 weeks before ovarian stimulation, the SARSCoV-2 IgM/IgG test can be used to decide to continue or postpone treatment until it is negative or negative. ASRM believes that the detection of SARS-CoV-2 antibodies is currently not part of the routine workflow of infertile patients, but the ESHRE believes this is critical.

In addition to COVID-19 infection from the provider, semen samples can become a potential source of infection. There may also be a 1-way infection. Four main areas of potential 2-way infection have been identified, i.e., patient-staff, staff-staff, staff-cell, and cell-cell. The results of a failure modes and effect analysis conducted by a multi- disciplinary IVF team showed that, except for cell-cell contamination, which is considered extremely unlikely, patient-employee, employee-employee, and employeecell interaction periods are estimated to have a moderate to high infection risk [50]. Therefore, routine SARSCoV-2 tests for patients and providers are necessary.

Because SARS-CoV-2 is an enveloped RNA virus, it can maintain its viability even at low temperatures, which can lead to cross-contamination between samples [51]. In assessing potential cross-contamination during cryopreservation and storage, the single recorded case of crosscontamination in tissue samples was hepatitis B in bone marrow, which was transmitted to the recipient [52]. The only known cross-contamination of tissues stored in IVF was experimental. Bielanski et al. [53] found that liquid nitrogen intentionally contaminated with bovine viral diarrhea virus spread to $21.3 \%$ of nearby open storage devices. However, all sealed straws and freezing tubes were not contaminated. In another similar study, mouse embryos were stored with murine virus vials and no crosscontamination was observed even after a year in storage [54]. These studies have shown that the risk of cross-contamination in liquid nitrogen is indeed negligible, especially when samples are stored and sealed properly. 
Due to the COVID-19 pandemic, medical institutions worldwide recommend performing surgical operations for only high-priority or emergency cases. Low priority cases, such as diagnostic semen analysis, sperm banks, elective surgery for sperm retrieval, and related fertility procedures, have been delayed for 6 months or longer and pose no increased health risks to individuals [55]. However, this pandemic may last longer than expected - potentially years. During this period, the average age of women trying to conceive will increase and ovarian reserve indicators will worsen. Contrastingly, male infertility is usually related to progressive testicular damage, such as varicocele. The adverse effects of delayed future ART treatments are a growing concern. With the suspension of treatments, patients feel more anxious and fearful of impairments in possible future pregnancies [56].

Studies have shown that patients with reduced ovarian reserves starting IVF treatment up to 180 days after the first visit will not have their pregnancies affected [57]. For high-risk patients with a poor response to ovarian stimulation, this observation still applies. Providers and patients should rest assured that, if short-term treatment needs to be delayed for medical, logistical, or economic reasons, the treatment results will not be affected [58]. When treating varicocele, surgery is not the only method. Only when progressive testicular growth retardation and/ or semen quality is severely impaired should clinical varicocele surgery be performed to maintain fertility [59].

Recently, 27 experts from 15 different countries expressed that postponing andrology services and male infertility treatments during the pandemic may permanently damage the chances of having children for "time-sensitive" patients [60]. This can have devastating psychological effects. The birth window for time-sensitive patients is short and any delay can be impactful. Such patients can be grouped into the following categories:

- Those with cancer and/or undergoing chemotherapy, radiotherapy, or immunosuppressive therapy

- Old age (>50 years)

- Those with severe male infertility (e.g., men with azoospermia/cryptospermia undergoing medical or postsurgical treatment to improve sperm quantity/quality)

- Those with inflammatory and systemic autoimmune diseases.

For such patients, the provision of andrology services cannot be regarded as a low priority [56]. Therefore, some experts believe that ART treatment can be carried out if the patient is considered high priority and has signed informed consent, agreeing to receive treatment and freezing procedures during a pandemic.

Challenges in Urology during the

COVID-19 Pandemic
Urological Diseases and COVID-19

Guidelines Office of the European Association of

Urology

The European Association of Urology (EAU) established a rapid reaction group after the virus outbreak to develop adaptive guidelines to deal with various situations and priorities. They divided urological diseases and conditions into the following 4 priority levels [55]:

- Low priority: clinical harm very unlikely if postponed for 6 months

- Intermediate priority: clinical harm possible, but unlikely, if postponed for 3-4 months

- High priority: clinical harm very likely if postponed for $>6$ weeks

- Emergency: life-threatening situation - cannot be postponed for $>24 \mathrm{~h}$.

Evidence from Wuhan shows that the mortality rate of asymptomatic patients who tested positive for COVID-19 after surgery was $20 \%$ [61]. Therefore, in the process of treating patients, doctors should choose the appropriate treatment plan according to the priority level. The latest guidelines provide some suggestions; for instance, surgery can be performed on high-priority and emergency patients during the COVID-19 pandemic, but surgery is not recommended for intermediate-priority patients. Next, we will introduce specific treatment plans for several common diseases in urology during the COVID-19 pandemic.

\section{Kidney Injury}

Since ACE2 is expressed in kidney cells, changes in the kidney due to COVID-19 need to have a point of focus [17]. Renal functioning needs to be monitored regularly, especially in patients with elevated plasma creatinine levels. In the event of early signs of acute kidney injury (AKI), interventions, such as continuous renal replacement therapy, should be implemented to protect renal functioning as early as possible. A study by Pei et al. [62] observed that COVID-19 patients have a high frequency of renal abnormalities, including $75.4 \%$ of patients with renal involvement, $65.8 \%$ with proteinuria, and $41.7 \%$ with hematuria.

The risk factors and causes of AKI in COVID-19 patients are diverse. The severity of pneumonia is the most important factor in the development of AKI in COVID-19 patients. The basic pathophysiology of patients with pneumonia is severe acute respiratory distress syndrome, which has been identified as an independent risk factor related to AKI [63]. This data indicates that it is necessary to provide in-depth support and careful moni- 
toring of COVID-19 patients with severe pneumonia to improve their complications. However, in a study of 116 COVID-19 hospitalized patients, Wang et al. [64] found that all patients without chronic kidney disease showed no obvious abnormality of renal function, and none of these patients showed AKI. Patients with chronic kidney disease who underwent CRRT were stable, without exacerbation of chronic kidney disease throughout the course of treatment of COVID-19.

\section{Renal Cancer}

The current COVID-19 pandemic is forcing medical staff to adapt their clinical practice, especially for the management of life-threatening diseases, such as malignant urological tumors. It is important to weigh the risk of contamination related to treatment and the risk of delaying treatment.

The CCAFU recommendations for the treatment of renal cancer during the COVID-19 pandemic are as follows [65]:

- Small T1a tumors, cystic tumors (Bosniak III and IV), and T1b tumors: surgery should be postponed under supervision

- CT2 tumors: surgery can be postponed under quarterly supervision

- T3 tumors, thrombus of the renal vein, vena cava thrombus, macroscopic lymphadenopathy without other secondary lesions, signs of locoregional invasion, and symptomatic tumors (pain, hematuria), for which no endoscopic or vascular treatment is possible: surgery must be maintained as a priority

- Indications of cytoreductive nephrectomy: favor according to the prognostic stage, monitoring, or medical treatment

- Metastatic renal cancer with a good prognosis: favor a tyrosine kinase inhibitor by encouraging telephone follow-up or teleconsultations

- Metastatic renal cancer with a poor or intermediate prognosis: the benefits of current standard treatments based on a ipilimumab-nivolumab combination must be weighed against the risk of severe toxicity in the context of reduced access to an intensive care unit. The use of sunitinib by default is suboptimal but can be discussed in this context. Patients who have an intermediate prognosis with a low tumor load and are asymptomatic can be treated with tyrosine kinase inhibitor. For patients with a poor prognosis and in poor general condition ( $P S \geq 2$ ), exclusive palliative treatment may be preferred.

\section{Bladder Cancer}

Patients with high-risk non-muscle-invasive bladder cancer need to be revisited to receive Calmette-Guérin Bacillus (BCG) intravesical instillation to reduce progression and recurrence after transurethral resection of the bladder [66]. It has been reported that nearly $30 \%$ of patients over 65 years of age may develop acute respiratory distress syndrome after infection with COVID-19 [66]. Most patients with non-muscle-invasive bladder cancer have a higher risk of severe forms of COVID-19 and may need to enter the intensive care unit for invasive ventilation because the median age of these patients is greater than 70 years [67].

In confirmed COVID-19 cases, due to the lack of data on the tolerance of intravesical BCG, the recommended and cautious approach is to delay additional instillation of BCG. Based on the infection status of COVID-19, it is recommended to delay the installation of BCG for at least 3 weeks after initial symptoms appear to improve. When dealing with side effects related to intravesical BCG infusion, attention should be paid to persistent fever by isolating and testing for COVID-19 [11]. Additionally, although side effects should be specifically treated according to current EAU guidelines, nonsteroidal antiinflammatory drugs should only be used in patients with no COVID-19 infection because these drugs may lead to a higher risk of hospitalization and intensive care unit admission [66].

\section{Prostate Cancer}

Surgery is recognized as the gold-standard treatment for several malignancies, including prostate cancer (PCa). With all healthcare systems and resources focused on controlling and treating COVID-19, oncologic surgery has been reduced worldwide. Surgery is associated with a higher overall and cancer-specific survival compared to radiation therapy alone. Apart from the theoretical advantage of radiation therapy in eliminating local micrometastases, clinical evidence shows that a real multimodal approach is best for patients choosing to undergo radical prostatectomy [68]. Delay in the detection or treatment of PCa can lead to impaired functional outcomes and higher rates of biochemical recurrence [69].

Surgery should only be performed in sterile COVID19-free facilities; otherwise, according to reports from Wuhan, postoperative mortality can reach $20 \%$ [61]. Unfortunately, the preservation of such facilities is impossible. According to the Italian experience, both asymptomatic caregivers and patients can be a source of infection, causing further spread within the hospital [70]. To
10

Urol Int 2021;105:3-16 DOI: $10.1159 / 000512880$
Jin/Park/Jung/Kim 
maintain a truly COVID-19-free facility, preoperative isolation and laboratory testing are needed to ensure that staff and patients have no infections.

Recently, studies have pointed out that there may be a potential link between PCa and COVID-19 [71]. A study showed that PCa patients who received androgen deprivation therapy had a 4-fold risk of SARS-CoV-2 infection than those who did not receive androgen deprivation therapy [71]. So far, there are 2 main genes related to the entry of the COVID-19 virus into host alveolar epithelial cells, i.e., ACE2 and TMPRSS2. The expression of TMPRSS2 is regulated by androgen/AR signaling. In primary and metastatic castration-resistant $\mathrm{PCa}$, the expressions of AR and TMPRSS2 were significantly positively correlated [72]. These findings indicate that more androgens may mean an increased expression of TMPRSS2, which may increase the susceptibility to SARS-CoV-2. Interestingly, a recent study showed that ACE2 expression is higher in men and may be regulated by androgen/AR signaling [73]. Therefore, androgen deprivation therapy can have a potential curative effect on COVID-19.

\section{Effects on the Testes}

The testes may be affected by COVID-19. A study reported that $19 \%$ of COVID-19 men suffer from scrotal discomfort, suggesting viral orchitis [74]. Orchitis has not been confirmed as a possible complication of SARS$\mathrm{CoV}-2$ infection. The virus may not directly infect the testes, but it may trigger a secondary autoimmune response that can cause autoimmune orchitis. COVID-19 is related to abnormal blood clotting, so orchitis could also be the result of segmental vasculitis. A recent study provided insight into impaired male gonadal function after COVID-19 infection [75]. That study showed that the testosterone-to-luteinizing hormone ratio in 81 patients with COVID-19 was dramatically decreased in comparison to 100 age-matched healthy counterparts [76]. The serum testosterone-to-luteinizing hormone ratio could be a potential marker of impairment of reproductive health caused by COVID-19. Another study also confirmed that the serum LH level of patients with COVID-19 was significantly higher than that of healthy men with normal fertility, while the ratio of serum testosterone toluteinizing hormone was significantly decreased, which is indicative of subclinical hypogonadism [77].

\section{Effects of the COVID-19 Pandemic on Urologists}

Outpatient Care

Due to the increased spread of the pandemic over time, in-person visits in the outpatient setting have decreased or even been canceled. The latest EAU guidelines recommend the implementation of telemedicine during this time [55]. The development of telemedicine has been a pragmatic approach to reducing the risk of further transmission. Nonemergency patients, such as those with follow-up tumors, benign conditions (such as moderate lower urinary tract symptom and prostate enlargement), and urinary tract infections, can be given a consultation over the telephone. Clinical priority should be given to patients with a suspected relapse or new malignant tumors, severe lower urinary tract syndromes, or potential obstructive/purulent urinary tract stones and those needing an immediate postoperative examination [78].

If a face-to-face interview is needed, both the provider and the patient need to wear masks and maintain social distancing [79]. Patients should also answer questions about their COVID-19-related exposure history or symptoms [80]. Patients with a positive screening are referred to isolated treatment. In the case of a negative screening, clinicians and patients must be provided with infection control procedures throughout the length of care.

Due to technological improvements and cost reductions as well as the widespread popularity of high-speed internet and smartphones, patients can quickly deploy telemedicine consultation from home [81]. The benefits of telemedicine include convenience, access to care from a distance, and lower medical costs. At the same time, it can reduce contact with patients and potential infection. Providers in quarantine or isolation can also continue to work [82]. One of the main disadvantages of telemedicine is that consumers lack awareness of its use, services, and costs. In addition, the lack of physical contact between patients and doctors also poses challenges when conducting remote physical examinations. Most countries lack a regulatory framework to authorize, integrate, and reimburse telemedicine care for all patients, especially in emergencies and outbreak situations [83].

When it comes to face-to-face medical consultations, the effects of facial coverings by masks can have a significant negative impact on patients [84]. The "distance" effect produced by maintaining social distancing requires a louder voice for communication, which in turn may harm patient privacy and reduce patient satisfaction [85].

\section{Urological Surgery}

Most benign urological surgeries, including surgeries for incontinence, benign prostatic hyperplasia, reconstruction, infertility, erectile dysfunction, and genitourinary prolapse [86], should be postponed until the pandemic is over. 
Ficarra et al. [86] and Stensland et al. [87] proposed excellent recommendations for urological practice during the COVID-19 pandemic. With regard to urinary tract obstruction or infection, ureteral stents or nephrostomy tubes under local anesthesia should be firstly considered. If this is not possible, stents under general anesthesia should be the next option. Acute urinary retention can be managed through insertion of a urethral or suprapubic catheter under local anesthesia. In cases of clot retention due to bladder cancer or $\mathrm{PCa}$, a cystoscopic evacuation should be considered and transurethral hemostasis of the tumor should be done to avoid the need for blood transfusions. Additionally, interventions should only be considered for pediatric urological emergencies, such as acute torsion (implement scrotal exploration and orchidopexy) and genitourinary obstruction (consider a Foley catheter and a suprapubic tube). In patients with a genitourinary trauma, surgical exploration is recommended only in hemodynamically unstable patients [87]; otherwise, procedures that can be performed with local anesthesia may be used. Lastly, the authors recommend immediate intervention for patients with refractory priapism (consider shunting), a scrotal abscess or Fournier gangrene (consider drainage and debridement, respectively), and an infected artificial urethral sphincter or penile prosthesis (removal of the infected device) $[86,87]$.

Aerosol transmission of COVID-19 cannot be ignored because it can survive in the environment for $3 \mathrm{~h}$ [88]. Because of this, it is recommended to use the lowest intraabdominal pressure on the pneumoperitoneum during robotic or laparoscopic surgery to reduce the risk of medical staff aerosol infection. Zheng et al. [89] recommend using lower power settings on electrocautery since ultrasonic scalpels or electrical devices may produce a large amount of surgical smoke. Additionally, adequate and complete deflation of the pneumoperitoneum may reduce the risk of infection. All console surgeons should wear goggles or sealed sunshades and carefully disinfect the console's head support between shells. Furthermore, it is recommended that endoscopic procedures and urethral catheterization be performed with caution, and surgeons should be completely protected against infection if the patient has suspected or confirmed COVID-19 [86].

In the latest EAU guidelines [55], there is some guidance on preoperative management, general surgery, and surgery for COVID-positive patients during the COVID pandemic. For example, in the preoperative stage, patients with clinical symptoms and/or those who have been in contact with COVID-19-positive individuals should receive a preoperative COVID-19 test. For patients without any clinical symptoms and no history of contact with COVID-19-positive patients, it is recommended that they undergo a COVID-19 test within $48 \mathrm{~h}$ before surgery. In general, medical staff should use complete PPE regardless of the patient's COVID-19 status. Patients also need to wear a full set of PPE if they are found to be positive for COVID-19. The operation should be performed by experienced surgeons and all unnecessary personnel should remain outside the operating room. Electrocautery devices should be used on reduced power settings to decrease the generation of surgical smoke. Flushing fluid should be collected through a closed system during the urological procedure. Elderly patients with comorbidities, even in high-priority cases, should be carefully considered for surgery. If a surgical patient is diagnosed with COVID-19, a special operating room must be prepared.

\section{Organ Transplantation in Urology}

Patients with end-stage kidney disease are at a higher risk of contracting infectious diseases due to their intrinsic fragility caused by their defective immune system [90]. Although there is no evidence that COVID-19 is transmitted through organ donations, this cannot be ignored because the virus has been detected in approximately $15 \%$ of patients [91]. The Transplant Association recommends COVID-19 testing of donors suspected of having the virus, coming from endemic areas, or having a history of possible contact [92]. Special care should be given solid organ transplant recipients as they are usually under chronic immunosuppression, which puts them at a higher risk of COVID-19 infection. On the other hand, the safety of the transplantation team is another concern; they can be exposed to transplant recipients who may potentially harbor higher viral loads than that of normal contact [91]. Therefore, the transplantation team must be cautious in areas with widespread community diffusion of COVID-19. In such situations, the US Organ Procurement and Transplantation Network suggests following $\mathrm{CDC}$ recommendations when evaluating suspected $\mathrm{CO}-$ VID-19 patients (mask the patient, place the patient in an isolation room, medical personnel must take airborne and contact precautions when contacting the patient, and inform the local authorities about the case) [93].

\section{Training for Urologists}

The COVID-19 pandemic has influenced residency training programs and medical graduate education. Most clinical rounds are canceled in many countries. Medical
Urol Int 2021;105:3-16 DOI: $10.1159 / 000512880$
Jin/Park/Jung/Kim 
training programs and teaching activities have switched to online platforms. For instance, in Singapore, interhospital staff movement among hospitals has been put on hold, while residents training in other hospitals will stay there indefinitely [94]. However, efforts are being made to ensure that these actions do not harm the long-term needs of medical trainees. Attention must be focused on how the COVID-19 pandemic and its effects, including the suspension of all nonemergency elective procedures, delay of inpatient examinations, and cessation of clinical rounds, may have a stressful impact on residents and students [95].

Clinical and Lab-Based Urological Research

During the first stages of the COVID-19 outbreak, many researchers focused on researching the treatment and prevention of the virus. Currently, there are no approved therapies or vaccines. Several national and international research groups are working collaboratively on a variety of preventative and therapeutic interventions. Some potential antiviral drugs, including nucleoside analogs, chloroquine, and protease inhibitors, are being urgently administered to patients with COVID-19 [3].

Favipiravir (T-705) is a guanine analog. A recent study suggested that favipiravir is a potential candidate for treating COVID-19, showing effective antiviral activities in Vero E6 cells [96]. Remdesivir (GS-5734) is an adenine analog. Remdesivir has emerged as the most promising candidate for the treatment of COVID-19 infection [96]. Both lopinavir and ritonavir are protease inhibitors and have been reported to have antiviral activities against SARS and MERS [97, 98]. For treatment of COVID-19, clinical trials (ChiCTR2000029539) have been initiated to test the antiviral activity of these protease inhibitors in patients [99]. However, the antiviral efficacy of HIV protease inhibitors in coronavirus proteases is controversial.

Vaccines are the most effective strategy for preventing infectious diseases because they are more cost-effective than treatments and can reduce morbidity and mortality without long-lasting effects. However, there are still no approved vaccines for human coronaviruses. Research groups around the world are accelerating the development of COVID-19 vaccines using various approaches, including vaccination based on subunit, DNA, and mRNA [100].

Clover Biopharmaceuticals is conducting preclinical testing of a recombinant subunit vaccine based on the trimeric S protein (S-Trimer) of SARS-CoV-2 [101]. The University of Queensland is developing subunit vaccines using the "molecular clamp," a transformative technology [102]. Inovio Pharmaceuticals, in collaboration with Beijing Advaccine Biotechnology, has started preclinical trials for a DNA vaccine (INO-4800) against SARSCoV-2 [103]. Moderna, Inc., has started phase I clinical trials for mRNA-1273, an mRNA vaccine, encoding the viral spike (S) protein of SARS-CoV-2 [104].

\section{Conclusions}

The COVID-19 pandemic is the biggest modern challenge facing the global healthcare system. After the pandemic, how will COVID-19 transform urological practice and research? Due to the limited supply of ventilators, manpower, and hospital resources, there is an urgent need to establish a new set of systems to meet demands in emergencies, such as replacing traditional outpatient services with telemedicine, reducing the number of nonnecessary operations, and implementing network teaching. In relation to urology, it is necessary to strengthen the safety training of medical staff. Since exposure to patients and bodily fluids presents potential increased risks of viral transmission, medical staff must be adequately protected. The concern of renal damage in patients from COVID-19 also requires much attention. Lastly, it is imperative to channel international resources into high-quality clinical trials with robust scientific rationale and vigorous statistical rigor to overcome this pandemic.

\section{Acknowledgement}

This research was supported by the Samuel Oschin Comprehensive Cancer Institute (SOCCI) at Cedars-Sinai Medical Center through the 2019 Lucy S. Gonda Award.

\section{Conflict of Interest Statement}

The authors have nothing to disclose.

\section{Funding Source}

This research was funded by the National Institutes of Health (1U01DK103260, 1R01DK100974, U24 DK097154, and NIH NCATS UCLA CTSI UL1TR000124), the Department of Defense (W81XWH-15-1-0415 and W81XWH-19-1-0109), the Centers for Disease Control and Prevention (1U01DP006079), and the USEgypt Science and Technology Development Fund by the National Academies of Sciences, Engineering, and Medicine (all to J.K.). 
This article is derived from the subject data funded in whole or part by the National Academies of Sciences, Engineering, and Medicine (NAS) and the United States Agency for International Development (USAID). Any opinions, findings, conclusions, or recommendations expressed in this article are those of the authors alone and do not necessarily reflect the views of the USAID or the NAS.

\section{Author Contributions}

Research conception and design: J.K. Data analysis and interpretation: P.J. and H.P. Drafting of this paper: P.J., S.J., and J.K. Supervision: J.K. Approval of the final version of this work: all of the authors.

\section{References}

1 Center for Systems Science Engineering at Johns Hopkins University. Coronavirus COVID-19 global cases [Internet].[cited 2020 Apr 3]. Available from: https://coronavirus. jhu.edu/map.html.

2 Masters PS. The molecular biology of coronaviruses. Adv Virus Res. 2006;66:193-292.

3 Ahn DG, Shin HJ, Kim MH, Lee S, Kim HS, Myoung J, et al. Current Status of Epidemiology, Diagnosis, Therapeutics, and Vaccines for Novel Coronavirus Disease 2019 (COVID-19). J Microbiol Biotechnol. 2020 Mar; 30(3):313-24.

4 Zhang W, Du RH, Li B, Zheng XS, Yang XL, $\mathrm{Hu} \mathrm{B}$, et al. Molecular and serological investigation of 2019-nCoV infected patients: implication of multiple shedding routes. Emerg Microbes Infect. 2020 Feb;9(1):386-9.

5 Ozma MA, Maroufi P, Khodadadi E, Kose S, Esposito I, Ganbarov K, et al. Clinical manifestation, diagnosis, prevention and control of SARS-CoV-2 (COVID-19) during the outbreak period. Infez Med. 2020 Jun;28(2):15365.

6 Tahamtan A, Ardebili A. Real-time RT-PCR in COVID-19 detection: issues affecting the results. Expert Rev Mol Diagn. 2020 May; 20(5):453-4.

7 Li Z, Yi Y, Luo X, Xiong N, Liu Y, Li S, et al. Development and clinical application of a rapid IgM-IgG combined antibody test for SARS-CoV-2 infection diagnosis. J Med Virol. 2020 Feb;92(9):1518-24.

8 Xie J, Ding C, Li J, Wang Y, Guo H, Lu Z, et al. Characteristics of patients with coronavirus disease (COVID-19) confirmed using an IgM-IgG antibody test. J Med Virol. 2020 Apr;92(10):2004-10.

9 US Food and Drug Administration. Emergency use authorizations for medical devices [Internet]. Available from: https://www.fda. gov/medical-devices/emergency-situationsmedical-devices/emergency-useauthorizations\#ivdnote2.

10 Chen N, Zhou M, Dong X, Qu J, Gong F, Han $\mathrm{Y}$, et al. Epidemiological and clinical characteristics of 99 cases of 2019 novel coronavirus pneumonia in Wuhan, China: a descriptive study. Lancet. 2020 Feb;395(10223):507-13.

11 Guan WJ, Ni ZY, Hu Y, Liang WH, Ou CQ, He JX, et al.; China Medical Treatment Expert Group for Covid-19. Clinical characteristics of coronavirus disease 2019 in China. N Engl J Med. 2020 Apr;382(18):1708-20.
12 Hamming I, Cooper ME, Haagmans BL, Hooper NM, Korstanje R, Osterhaus AD, et al. The emerging role of ACE2 in physiology and disease. J Pathol. 2007 May;212(1):1-11.

$13 \mathrm{Xu} \mathrm{X}$, Chen P, Wang J, Feng J, Zhou H, Li X, et al. Evolution of the novel coronavirus from the ongoing Wuhan outbreak and modeling of its spike protein for risk of human transmission. Sci China Life Sci. 2020 Mar;63(3): 457-60.

14 Hoffmann M, Kleine-Weber H, Schroeder S, Krüger N, Herrler T, Erichsen S, et al. SARSCoV-2 Cell Entry Depends on ACE2 and TMPRSS2 and Is Blocked by a Clinically Proven Protease Inhibitor. Cell. $2020 \mathrm{Apr}$ 181(2): 271-280.e8.

15 Li Y, Zhou W, Yang L, You R. Physiological and pathological regulation of ACE2, the SARS-CoV-2 receptor. Pharmacol Res. 2020 Jul; 157:104833.

16 Paoloni-Giacobino A, Chen H, Peitsch MC, Rossier C, Antonarakis SE. Cloning of the TMPRSS2 gene, which encodes a novel serine protease with transmembrane, LDLRA, and SRCR domains and maps to 21q22.3. Genomics. 1997 Sep;44(3):309-20.

17 Dong M, Zhang J, Ma X, Tan J, Chen L, Liu S, et al. ACE2, TMPRSS2 distribution and extrapulmonary organ injury in patients with $\mathrm{CO}$ VID-19. Biomed Pharmacother. 2020 Nov; 131:110678.

18 Matsuyama S, Nao N, Shirato K, Kawase M, Saito S, Takayama I, et al. Enhanced isolation of SARS-CoV-2 by TMPRSS2-expressing cells. Proc Natl Acad Sci USA. 2020 Mar; 117(13):7001-3.

19 Stopsack KH, Mucci LA, Antonarakis ES, Nelson PS, Kantoff PW. TMPRSS2 and COVID-19: Serendipity or Opportunity for Intervention? Cancer Discov. 2020 Jun;10(6): 779-82.

20 Chen Y, Guo Y, Pan Y, Zhao ZJ. Structure analysis of the receptor binding of 2019nCoV. Biochem Biophys Res Commun. 2020 Feb;525(1):S0006-291X(20)30339-9.

21 Paoli D, Pallotti F, Colangelo S, Basilico F, Mazzuti L, Turriziani O, et al. Study of SARSCoV-2 in semen and urine samples of a volunteer with positive naso-pharyngeal swab. J Endocrinol Invest. 2020 Dec;43(12):1819-22.

22 Song C, Wang Y, Li W, Hu B, Chen G, Xia P, et al. Absence of 2019 novel coronavirus in semen and testes of COVID-19 patients. Biol Reprod. 2020 Apr;103(1):4-6.
23 Holtmann N, Edimiris P, Andree M, Doehmen C, Baston-Buest D, Adams O, et al. Assessment of SARS-CoV-2 in human semen-a cohort study. Fertil Steril. 2020 Aug;114(2):233-8.

24 Li D, Jin M, Bao P, Zhao W, Zhang S. Clinical Characteristics and Results of Semen Tests Among Men With Coronavirus Disease 2019. JAMA Netw Open. 2020 May;3(5):e208292.

25 Zhang S, Wang X, Zhang H, Xu A, Fei G, Jiang $\mathrm{X}$, et al. The absence of coronavirus in expressed prostatic secretion in COVID-19 patients in Wuhan city. Reprod Toxicol. 2020 Jun;96:90-4.

26 Global Health 5050. COVID-19 sex-disaggregated data tracker [Internet]. 2020. Available from: http://globalhealth5050.org/covid19.

27 Lipsky MS, Hung M. Men and COVID-19: A pathophysiologic review. Am J Men Health. 2020 Sep-Oct; 14(5):1557988320954021.

28 Karlberg J, Chong DS, Lai WY. Do men have a higher case fatality rate of severe acute respiratory syndrome than women do? Am J Epidemiol. 2004 Feb;159(3):229-31.

29 Cascella M, Rajnik M, Cuomo A, Dulebohn SC, Di Napoli R. Features, Evaluation, and Treatment of Coronavirus (COVID-19). Treasure Island (FL): StatPearls; 2020.

30 Schurz H, Salie M, Tromp G, Hoal EG, Kinnear CJ, Möller M. The X chromosome and sex-specific effects in infectious disease susceptibility. Hum Genomics. 2019 Jan; 13(1):2.

31 Klein SL, Marriott I, Fish EN. Sex-based differences in immune function and responses to vaccination. Trans R Soc Trop Med Hyg. 2015 Jan;109(1):9-15.

32 Salonia A, Corona G, Giwercman A, Maggi M, Minhas S, Nappi RE, et al. SARS-CoV-2, testosterone and frailty in males (PROTEGGIMI): A multidimensional research project. Andrology. 2020 May;andr.12811.

33 Robinson DP, Hall OJ, Nilles TL, Bream JH, Klein SL. 17 $\beta$-estradiol protects females against influenza by recruiting neutrophils and increasing virus-specific CD8 T cell responses in the lungs. J Virol. 2014 May;88(9): 4711-20.

34 Suba Z. Prevention and therapy of COVID-19 via exogenous estrogen treatment for both male and female patients. J Pharm Pharm Sci. 2020;23(1):75-85.

35 Suen LK, So ZY, Yeung SK, Lo KY, Lam SC. Epidemiological investigation on hand hygiene knowledge and behaviour: a cross-sectional study on gender disparity. BMC Public Health. 2019 Apr;19(1):401. 
36 Chu DK, Akl EA, Duda S, Solo K, Yaacoub S, Schünemann HJ, et al.; COVID-19 Systematic Urgent Review Group Effort (SURGE) study authors. Physical distancing, face masks, and eye protection to prevent personto-person transmission of SARS-CoV-2 and COVID-19: a systematic review and metaanalysis. Lancet. 2020 Jun;395(10242):197387.

37 G C. Bulk and single-cell transcriptomics identify tobacco-use disparity in lung gene expression of ACE2, the receptor of 2019nCov. medRxiv. 2020.

$38 \mathrm{Yu}$ J, Yu J, Mani RS, Cao Q, Brenner CJ, Cao $\mathrm{X}$, et al. An integrated network of androgen receptor, polycomb, and TMPRSS2-ERG gene fusions in prostate cancer progression. Cancer Cell. 2010 May;17(5):443-54.

39 Souza MD, Nakagawa H, Taitson PF, Cordts EB, Antunes RA. Management of ART and COVID-19: infertility in times of pan- demic. What now? JBRA Assist Reprod. 2020 Jul; 24(3):231-2.

40 Liang H, Acharya G. Novel corona virus disease (COVID-19) in pregnancy: what clinical recommendations to follow? Acta Obstet Gynecol Scand. 2020 Apr;99(4):439-42.

41 World Health Organization. World Health Organization report of the WHO-China Joint Mission on Coronavirus Disease 2019 (COVID-19) [Internet]. 2020. Available from: https://wwwwhoint/docs/default-source/ coronaviruse/who-china-joint-mission-oncovid-19-final-reportpdf.

42 Wang S, Guo L, Chen L, Liu W, Cao Y, Zhang J, et al. A Case Report of Neonatal 2019 Coronavirus Disease in China. Clin Infect Dis. 2020 Jul;71(15):853-7.

43 Maleki Dana P, Kolahdooz F, Sadoughi F, Moazzami B, Chaichian S, Asemi Z. COVID-19 and pregnancy: a review of current knowledge. Infez Med. 2020 Jun;28 suppl 1: 46-51.

44 European Society of Human Reproduction and Embryology. ESHRE statement on pregnancy and conception [Internet]. 2020. Available from: https://wwweshreeu/Press-Room/ ESHRE-News\#CoronaStatement27feb.

45 European Society of Human Reproduction and Embryology. Safe ART services during the third phaseof the COVID-19 pandemic [Internet]. Available from: https://www.eshre.eu/-/media/sitecore-files/Guidelines/ COVID19/ESHRE-guidance_Safe-ART-services-during-the-third-phase-of-the-COVID19-pandemic.pdf.

46 American Society for Reproductive Medicine. American Society for Reproductive Medicine patient management and clinical recommendations during the coronavirus (COVID-19) pandemic [Internet]. 2020. Available from: https://www.asrm.org/news-and-publications/covid-19/statements/patient-management-and-clinical-recommendations-during-the-coronavirus-covid-19-pandemic.
47 American Society for Reproductive Medicine. American Society for Reproductive Medicine patient management and clinical recommendations during the coronavirus (COVID-19) pandemic: update \#5 [Internet]. Available from: https://www.asrm.org/globalassets/ asrm/asrm-content/news-and-publications/ covid-19/covidtaskforceupdate5.pdf.

48 De Paoli P. Bio-banking in microbiology: from sample collection to epidemiology, diagnosis and research. FEMS Microbiol Rev. 2005 Nov;29(5):897-910.

49 La Marca A, Nelson SM. SARS-CoV-2 testing in infertile patients: different recommendations in Europe and America. J Assist Reprod Genet. 2020 Aug;37(8):1823-8.

50 Maggiulli R, Giancani A, Fabozzi G, Dovere L, Tacconi L, Amendola MG, et al. Assessment and management of the risk of SARSCoV-2 infection in an IVF laboratory. Reprod Biomed Online. 2020 Sep;41(3):385-94.

51 Bielanski A. A review of the risk of contamination of semen and embryos during cryopreservation and measures to limit cross-contamination during banking to prevent disease transmission in ET practices. Theriogenology. 2012 Feb;77(3):467-82.

52 Tedder RS, Zuckerman MA, Goldstone AH, Hawkins AE, Fielding A, Briggs EM, et al. Hepatitis B transmission from contaminated cryopreservation tank. Lancet. 1995 Jul;346 (8968):137-40.

53 Bielanski A, Nadin-Davis S, Sapp T, LutzeWallace C. Viral contamination of embryos cryopreserved in liquid nitrogen. Cryobiology. 2000 Mar;40(2):110-6.

54 Kyuwa S, Nishikawa T, Kaneko T, Nakashima T, Kawano K, Nakamura N, et al. Experimental evaluation of cross-contamination between cryotubes containing mouse 2-cell embryos and murine pathogens in liquid nitrogen tanks. Exp Anim. 2003 Jan;52(1):67-70.

55 Ribal MJ, Cornford P, Briganti A, Knoll T, Gravas S, Babjuk M, et al.; GORRG Group; EAU Section Offices and the EAU Guidelines Panels. European Association of Urology Guidelines Office Rapid Reaction Group: An Organisation-wide Collaborative Effort to Adapt the European Association of Urology Guidelines Recommendations to the Coronavirus Disease 2019 Era. Eur Urol. 2020 Jul;78(1):21-8.

56 Esteves SC, Lombardo F, Garrido N, Alvarez J, Zini A, Colpi GM, et al. SARS-CoV-2 pandemic and repercussions for male infertility patients: A proposal for the individualized provision of andrological services. Andrology. 2020 May;andr.12809.

57 Romanski PA, Bortoletto P, Rosenwaks Z, Schattman GL. Delay in IVF treatment up to 180 days does not affect pregnancy outcomes in women with diminished ovarian reserve. Hum Reprod. 2020 Jul;35(7):1630-6.

58 Reřuchová $M$, Březinová J, Filipčíková $R$, Oborná I. [Influence of the length of cultivation of no early cleavage embryos on the IVF success rate]. Ceska Gynekol. 2013 Jan;78(1): $68-72$.
59 Jensen CF, Østergren P, Dupree JM, Ohl DA, Sønksen J, Fode M. Varicocele and male infertility. Nat Rev Urol. 2017 Sep;14(9):523-33.

60 Hallak J, Esteves SC. Concise practice recommendations for the provision of andrological services and assisted reproductive technology for male infertility patients during the SARSCoV-2 in Brazil. Int Braz J Urol. 2020 NovDec;46(6):1082-9.

61 Lei S, Jiang F, Su W, Chen C, Chen J, Mei W, et al. Clinical characteristics and outcomes of patients undergoing surgeries during the incubation period of COVID-19 infection. EClinicalMedicine. 2020 Apr;21:100331.

62 Pei G, Zhang Z, Peng J, Liu L, Zhang C, Yu C, et al. Renal Involvement and Early Prognosis in Patients with COVID-19 Pneumonia. J Am Soc Nephrol. 2020 Jun;31(6):1157-65.

63 Darmon M, Clec'h C, Adrie C, Argaud L, Allaouchiche B, Azoulay E, et al. Acute respiratory distress syndrome and risk of AKI among critically ill patients. Clin J Am Soc Nephrol. 2014 Aug;9(8):1347-53.

64 Wang L, Li X, Chen H, Yan S, Li D, Li Y, et al. Coronavirus Disease 19 Infection Does Not Result in Acute Kidney Injury: An Analysis of 116 Hospitalized Patients from Wuhan, China. Am J Nephrol. 2020;51(5):343-8.

65 Méjean A, Rouprêt M, Rozet F, Bensalah K, Murez T, Game X, et al.; le comité de cancérologie de l'Association française d'urologie (CCAFU). [Recommendations CCAFU on the management of cancers of the urogenital system during an epidemic with Coronavirus COVID-19]. Prog Urol. 2020 Apr;30(5):22131.

66 Lenfant L, Seisen T, Loriot Y, Rouprêt M. Adjustments in the Use of Intravesical Instillations of Bacillus Calmette-Guérin for Highrisk Non-muscle-invasive Bladder Cancer During the COVID-19 Pandemic. Eur Urol. 2020 Jul;78(1):1-3.

67 American Cancer Society. Cancer Facts \& Figures 2020. Atlanta: ACA; 2020.

68 Wang Z, Ni Y, Chen J, Sun G, Zhang X, Zhao $J$, et al. The efficacy and safety of radical prostatectomy and radiotherapy in high-risk prostate cancer: a systematic review and metaanalysis. World J Surg Oncol. 2020 Feb;18(1): 42.

69 Moschovas MC, Sighinolfi MC, Rocco B, Bhat S, Onol F, Rogers T, et al. Balancing the Effects of COVID-19 Against Potential Progression and Mortality in High-risk Prostate Cancer. Eur Urol. 2020 Jul;78(1):e14-5.

70 Coccolini F, Perrone G, Chiarugi M, Di Marzo F, Ansaloni L, Scandroglio I, et al. Surgery in COVID-19 patients: operational directives. World J Emerg Surg. 2020 Apr;15(1):25.

71 Montopoli M, Zumerle S, Vettor R, Rugge M, Zorzi M, Catapano CV, et al. Androgen-deprivation therapies for prostate cancer and risk of infection by SARS-CoV-2: a population-based study $(\mathrm{N}=4532)$. Ann Oncol. 2020 Aug;31(8):1040-5. 
72 Lucas JM, Heinlein C, Kim T, Hernandez SA, Malik MS, True LD, et al. The androgen-regulated protease TMPRSS2 activates a proteolytic cascade involving components of the tumor microenvironment and promotes prostate cancer metastasis. Cancer Discov. 2014 Nov;4(11):1310-25.

73 Wei X, Xiao YT, Wang J, Chen R, Zhang W, Yang $Y$, et al. Sex differences in severity and mortality among patients with COVID-19: evidence from pooled literature analysis and insights from integrated bioinformatic analysis. 2020. doi: arXiv:2003.13547.

74 Pan F, Xiao X, Guo J, Song Y, Li H, Patel DP, et al. No evidence of severe acute respiratory syndrome-coronavirus 2 in semen of males recovering from coronavirus disease 2019. Fertil Steril. 2020 Jun;113(6):1135-9.

75 Wang S, Zhou X, Zhang T, Wang Z. The need for urogenital tract monitoring in COVID-19. Nat Rev Urol. 2020 Jun;17(6):314-5.

$76 \mathrm{Ma} \mathrm{L}$ ea. Effect of SARS-CoV-2 infection upon male gonadal function: a single centerbased study. medRxiv. 2020.

77 Pozzilli P, Lenzi A. Commentary: Testosterone, a key hormone in the context of COVID-19 pandemic. Metabolism. 2020 Jul;108: 154252.

78 Puliatti S, Eissa A, Eissa R, Amato M, Mazzone E, Dell'Oglio P, et al. COVID-19 and urology: a comprehensive review of the literature. BJU Int. 2020 Jun;125(6):E7-14.

79 Lotfi M, Hamblin MR, Rezaei N. COVID-19: Transmission, prevention, and potential therapeutic opportunities. Clin Chim Acta. 2020 Sep;508:254-66.

80 Luciani LG, Mattevi D, Cai T, Giusti G, Proietti S, Malossini G. Teleurology in the time of Covid-19 pandemic: here to stay? Urology. 2020 Jun;140:4-6.

81 Ohannessian R, Duong TA, Odone A. Global Telemedicine Implementation and Integration Within Health Systems to Fight the COVID-19 Pandemic: A Call to Action. JMIR Public Health Surveill. 2020 Apr;6(2):e18810.

82 Miller A, Rhee E, Gettman M, Spitz A. The Current State of Telemedicine in Urology. Med Clin North Am. 2018 Mar;102(2):38798.

83 Smith AC, Thomas E, Snoswell CL, Haydon $\mathrm{H}$, Mehrotra A, Clemensen J, et al. Telehealth for global emergencies: implications for coronavirus disease 2019 (COVID-19). J Telemed Telecare. 2020 Jun;26(5):309-13.

84 Wong CK, Yip BH, Mercer S, Griffiths S, Kung K, Wong MC, et al. Effect of facemasks on empathy and relational continuity: a randomised controlled trial in primary care. BMC Fam Pract. 2013 Dec;14(1):200.
85 Ghosh A, Sharma K, Choudhury S. COVID-19 and physician-patient relationship: potential effects of 'masking', 'distancing' and 'others'. Fam Pract. 2020 Sep;cmaa092.

86 Ficarra V, Novara G, Abrate A, Bartoletti R, Crestani A, De Nunzio C, et al.; Research Urology Network (RUN). Urology practice during the COVID-19 pandemic. Minerva Urol Nefrol. 2020 Jun;72(3):369-75.

87 Stensland KD, Morgan TM, Moinzadeh A, Lee CT, Briganti A, Catto JW, et al. Considerations in the Triage of Urologic Surgeries During the COVID-19 Pandemic. Eur Urol. 2020 Jun;77(6):663-6.

88 van Doremalen N, Bushmaker T, Morris DH, Holbrook MG, Gamble A, Williamson BN, et al. Aerosol and surface stability of SARS$\mathrm{CoV}-2$ as compared with SARS-CoV-1. N Engl J Med. 2020 Apr;382(16):1564-7.

89 Zheng MH, Boni L, Fingerhut A. Minimally Invasive Surgery and the Novel Coronavirus Outbreak: lessons Learned in China and Italy. Ann Surg. 2020 Jul;272(1):e5-6.

90 Xiao Y, Qian K, Luo Y, Chen S, Lu M, Wang G, et al. Severe Acute Respiratory Syndrome Coronavirus 2 Infection in Renal Failure Patients: A Potential Covert Source of Infection. Eur Urol. 2020 Aug;78(2):298-9.

91 Kumar D, Manuel O, Natori Y, Egawa H, Grossi P, Han SH, et al. COVID-19: A global transplant perspective on successfully navigating a pandemic. Am J Transplant. $2020 \mathrm{Jul}$; 20(7):1773-9.

92 The Transplantation Society. An update and guidance on 2019 novel coronavirus (COVID-19) for transplant clinicians. Available from: https://tts.org/23-tid/tid-news/657-tidupdate-and-guidance-on-2019-novel-coronavirus-2019-ncov-for-transplant-id.

93 US Department of Health and Human Services. Information for transplant programs and OPOs regarding 2019 novel coronavirus: OPTN [Internet]. 2019. [cited 2020 Mar]. Available from: https://optn.transplant.hrsa. gov/news/information-for-transplant-programs-and-opos-regarding-2019-novelcoronavirus/.

94 Chan MC, Yeo SE, Chong YL, Lee YM. Stepping Forward: Urologists' Efforts During the COVID-19 Outbreak in Singapore. Eur Urol. 2020 Jul;78(1):e38-9.

95 Rimmer A. Trainees and COVID-19: your questions answered. BMJ. 2020 Mar; 368:m1059.

96 Wang M, Cao R, Zhang L, Yang X, Liu J, Xu $M$, et al. Remdesivir and chloroquine effectively inhibit the recently emerged novel coronavirus (2019-nCoV) in vitro. Cell Res. 2020 Mar;30(3):269-71.
97 Chan KS, Lai ST, Chu CM, Tsui E, Tam CY, Wong MM, et al. Treatment of severe acute respiratory syndrome with lopinavir/ritonavir: a multicentre retrospective matched cohort study. Hong Kong Med J. 2003 Dec; 9(6):399-406.

98 Chu CM, Cheng VC, Hung IF, Wong MM, Chan KH, Chan KS, et al.; HKU/UCH SARS Study Group. Role of lopinavir/ritonavir in the treatment of SARS: initial virological and clinical findings. Thorax. 2004 Mar;59(3): 252-6.

99 Lythgoe MP, Middleton P. Ongoing Clinical Trials for the Management of the COVID-19 Pandemic. Trends Pharmacol Sci. 2020 Jun; 41(6):363-82.

100 Wang J, Peng Y, Xu H, Cui Z, Williams RO 3rd. The COVID-19 Vaccine Race: Challenges and Opportunities in Vaccine Formulation. AAPS PharmSciTech. 2020 Aug, 21(6):225.

101 Clover Biopharmaceuticals. Vaccines programs. Available from: http://www.cloverbiopharma.com/index.php? $\mathrm{m}=$ content $\& \mathrm{c}=$ index\&a=lists\&catid $=42$.

102 The University of Queensland Australia. 'Significant step' in COVID-19 vaccine quest [Internet]. [cited $2020 \mathrm{Feb}$ 28]. Available from: https://www.uq.edu.au/news/article/2020/02/significantstep\%E2\%80\%99covid-19-vaccine-quest.

103 INOVIO. INOVIO accelerates timeline for COVID-19 DNA vaccine INO-4800 [Internet]. [cited 2020 Mar 3]. Available from: http://ir.inovio.com/news-andmedia/news/ press-release-details/2020/Inovio-Accelerates-Timeline-for-COVID-19-DNA-Vaccine-INO-4800/default.aspx.

104 Moderna. Moderna's pipeline [Internet]. [cited 2020 Feb 28]. Available from: https:// www.modernatx.com/pipeline.

105 Zhen W, Smith E, Manji R, Schron D, Berry GJ. Clinical Evaluation of Three Sample-toAnswer Platforms for Detection of SARSCoV-2. J Clin Microbiol. 2020 Jul; 58(8):e00783-20.

106 GenMark Diagnostics. ePlex ${ }^{\circledR}$ SARS-CoV-2 test assay manual. Available from: https:// www.fda.gov/media/136282/download.

107 Seegene. AllplexTM 2019-nCoV assay: version 2.1; October 30th, 2020. Available from: https://www.fda.gov/media/137178/download.

108 Hardy Diagnostics. Instructions for use: Anti-SARS-CoV-2 rapid test. Available from: https://www.fda.gov/media/137367/download. 